\title{
MicroRNA expression profiles in human breast cancer cells after multifraction and single-dose radiation treatment
}

\author{
CHUNG-MAN LEUNG ${ }^{1,2}$, TING-WEN CHEN ${ }^{3,4}$, SUNG-CHOU LI $^{5}$, MENG-RU HO ${ }^{6}$, LING-YUEH HU ${ }^{7}$, \\ WEN-SHAN LIU ${ }^{1}$, TONY T. WU ${ }^{8,9}$, PING-CHI HSU ${ }^{2}$, HONG-TAI CHANG ${ }^{8,10}$ and KUO-WANG TSAI ${ }^{11}$ \\ ${ }^{1}$ Department of Radiation Oncology, Kaohsiung Veterans General Hospital, Kaohsiung; ${ }^{2}$ Department of Safety, \\ Health and Environmental Engineering, National Kaohsiung First University of Science and Technology, Kaohsiung; \\ ${ }^{3}$ Molecular Medicine Research Center and ${ }^{4}$ Bioinformatics Center, Chang Gung University, Taoyuan; \\ ${ }^{5}$ Genomics and Proteomics Core Laboratory, Department of Medical Research, Kaohsiung Chang Gung \\ Memorial Hospital and Chang Gung University College of Medicine, Kaohsiung; ${ }^{6}$ Biodiversity Research Center and \\ ${ }^{7}$ Institute of Biomedical Sciences, Academia Sinica, Taipei; ${ }^{8}$ Department of Surgery, Kaohsiung Veterans General Hospital, \\ Kaohsiung; ${ }^{9}$ School of Medicine, Yang-Ming University, Taipei; Departments of ${ }^{10}$ Emergency and \\ ${ }^{11}$ Medical Education and Research, Kaohsiung Veterans General Hospital, Kaohsiung, Taiwan, R.O.C.
}

Received January 7, 2014; Accepted February 27, 2014

DOI: $10.3892 /$ or.2014.3089

\begin{abstract}
MicroRNAs (miRNAs) are small non-coding RNAs that contribute to modulating signaling pathways after radiation exposure and have emerged as a potential therapeutic target or biomarker in the radiation response of cancer. Exposing breast cancer cells to single-dose (SD) or multifractionated (MF) radiation may affect the cells differently. However, the roles of miRNAs in breast cancer cells after the response to SD or $\mathrm{MF}$ is not thoroughly understood. Therefore, the purpose of the present study was to comprehensively investigate the response of miRNAs in MDA-MB-361 by using various radiation exposing protocols. Our results revealed that only a small fraction of miRNAs exhibiting differential expressions ( $>1.5$-fold) was identified after MDA-MB-361 cells were exposed to SD (10 Gy) or $\mathrm{MF}$ radiation (2 Gy x $5 \mathrm{MF}$ ). In addition, we observed that several miRNAs in the MDA-MB-361 cells frequently exhibited differential responses to various types of radiation treatment. Among these miRNAs, the expression levels of an oncogenic miR-17-92 cluster increased following SD radiation treatment. Conversely, miR-19a-3p, miR-20a-5p, and miR-19b-3p expressions were inhibited by $>1.5$-fold in the following MF treatment. Further analysis of the miR-17-92 cluster expression levels revealed that miR-17, miR-18a, miR-19a/b and miR-20a were significantly overexpressed and miR-92a was downregulated in
\end{abstract}

Correspondence to: Professor Kuo-Wang Tsai, Department of Medical Education and Research, Kaohsiung Veterans General Hospital, Kaohsiung 813, Taiwan, R.O.C.

E-mail:kwtsai@vghks.gov.tw; kwtsai6733@gmail.com

Dr Hong-Tai Chang, Department of Surgery, Kaohsiung Veterans General Hospital, Kaohsiung 813, Taiwan, R.O.C.

E-mail: htchang@vghks.gov.tw

Key words: microRNA, breast cancer, next-generation sequencing, pathway enrichment analysis, radiation, TCGA, miR-17-92 cluster breast cancer. Functional annotation demonstrated that target genes of the miR-17-92 cluster were predominantly involved in the regulation of radiation-associated signal pathways such as mitogen-activated protein kinase (MAPK), ErbB, p53, Wnt, transforming growth factor- $\beta$ (TGF- $\beta$ ), mTOR signaling pathways and cell cycles with an FDR $<0.05$. Overall, the results of the present study revealed distinct differences in the response of miRNAs to SD and MF radiation exposure, and these radiationassociated miRNAs may contribute to radiosensitivity and can be used as biomarkers for radiotherapy.

\section{Introduction}

Ionizing radiation, used alone or in combination with surgery and chemotherapy, plays an essential role in the management of breast cancer from the early to advanced stages. More than half of all patients with breast cancer are treated with radiotherapy (1). Ionizing radiation damages cells by using free radicals from the radiolysis of water that cause DNA double-strand breaks. Extensive biological effects are induced in the cells after radiation exposure, including apoptosis and DNA repair $(2,3)$. A complex cell response is elicited to repair radiation damage, including an alteration in gene expression, particularly in genes involved in stress response, cell cycle control and DNA repair (4). Radiation sensitivity varies widely among people, and much of the difference in the effects of radiation exposure is observed at the gene expression level (5). A more comprehensive understanding of the tumor radiationrelated genes can be particularly useful for predicting tumor response to radiotherapy and potentially modulating the treatment outcome of breast cancer patients.

Clinically, fractionation is widely used in radiotherapy with multiple 2-Gy fractions over the course of several weeks with total radiation doses of 50-60 Gy. This process allows for sufficient time between dose fractions, it provides treatment benefits involving the further sparing of normal tissue by 
repairing the sublethal damage, and it increases the damage to the tumor through the reoxygenation and reassortment of cells into radiosensitive phases of the cycle between dose fractions. However, in recent years, radiotherapy involving large fraction size [including stereotactic body radiation therapy (SBRT)] and doses from 5 Gy to 25 Gy per fraction has become a mainstay for treating lung cancer or metastatic tumors to achieve more effective tumor control. Tsai et al (6) determined that, at the molecular level, the patterns of gene expression vary substantially between single-dose (SD) and fractionated radiation in 3 cancer cell lines, which results in the differential expression of 463 genes, of which 13 are commonly upregulated in MCF7, SF539 and DU145 cells. Compared with SD radiation, another study revealed that a more robust induction of genes occurs during fractionated radiation (7).

MicroRNAs (miRNAs) are small RNAs of $\sim 22$ nucleotides in length that act as crucial negative regulators of protein expression at the post-transcriptional level (8). These molecules function as inhibitors of target mRNA for either the degradation or inhibition of gene function. Researchers have suggested that miRNAs are responsible for controlling $\sim 50 \%$ of all proteincoding genes (9). Studies have indicated that the expression of miRNAs has been clearly involved in cancer development, and the alternation of miRNAs has been observed in various types of cancer, including breast cancer. Iorio et al (10) identified miRNA aberrant expression in human breast cancer by using systematic profiling, and revealed that it is specifically correlated with pathological features of breast cancer, such as estrogen and progesterone receptor expression, tumor stage, vascular invasion and cell proliferation. miRNAs have also been determined to play vital roles in a majority of biological processes in breast cancer, including tumor cell growth, apoptosis, invasion and metastasis. miR-125a suppresses cell growth and induces apoptosis in breast cancer cells by targeting the mRNA encoding the RNA-stabilizing protein HuR (11). In a murine xenograft model study, invasion and metastasis were induced by the overexpression of miR-10b in a breast cancer tumor (12). Among these dysfunctional miRNAs, several have been demonstrated to play a critical role in breast cancer radiosensitivity, including let-7 family miR-7, miR-21, miR-31, miR-200c, miR-199a and miR-302a (13-18). However, these radioresponse miRNA expressions may differ depending on whether cells are exposed to an SD or multifractionated (MF) radiation dose. In the present study, miRNA expression profiles were compared in human MDA-MB-361 cells exposed to an SD or MF radiation dose by using the next-generation sequence approach. The data revealed that differential expression patterns of miRNAs occurred when using 2 distinct radiation protocols. Among these patterns, the response of miR-17-92 cluster expression clearly differed between the SD and MF radiation dose in MDA-MB-361 cells. We further investigated the role of the miR-17-92 cluster in breast cancer by using in silico analysis. We concluded that these radiation-response miRNAs can be used as therapeutic targets for improving the efficacy of radiation treatment in future breast cancer therapy.

\section{Materials and methods}

Cell culture and radiation treatment. Breast cancer cells, MDA-MB-361, were obtained from the American Type
Culture Collection and were maintained in Dulbecco's modified Eagle's medium, supplemented with $10 \%$ inactivated fetal bovine serum (FBS; Invitrogen, Carlsbad, CA, USA). The cells were exposed to various radiation dosages $(0,2,6,10,14$ and $18 \mathrm{~Gy}$ ), and were subsequently cultured in fresh medium. The total RNA was obtained at $15 \mathrm{~h}$ following radiation treatment, using TRIzol (Invitrogen) according to the manufacturer's instructions. The concentration, purity and amount of total RNA were determined using a NanoDrop 1000 spectrophotometer (NanoDrop Technologies Inc., USA).

Clinical breast cancer samples. Breast cancer samples (including 44 breast tumors and 29 adjacent normal tissues) were collected from 44 breast cancer patients who received a surgical operation at the Department of Surgery, Kaohsiung Veterans General Hospital. Informed consent was obtained from all the patients. The total RNA of the tissue was extracted using a TRIzol reagent, according to the instructions.

Collection and preprocessing of sequence reads. The MDA-MB-361 cells were exposed to $10 \mathrm{~Gy}$ of radiation, administered either as SD or MF radiation. To administer MF radiation, the cells were exposed to $2 \mathrm{~Gy}$ a day for 5 days. After the final dose of radiation treatment was administered, the cells were lysed at $15 \mathrm{~h}$ for RNA extraction. The RNA samples were prepared using an Illumina small RNA preparation kit, and were subsequently sequenced using the Illumina HiSeq platform. The generated sequence reads were first subjected to quality control to remove low-quality reads. The sequence reads were then subjected to $3^{\prime}$ adaptor trimming to generate clean reads, as previously described $(19,20)$. To attain a high confidence level, only the clean reads with a read count $\geq 2$ and with a length ranging from 15 to $27 \mathrm{nt}$ were included in further analyses.

Mapping clean reads to pre-miRNAs. To investigate the miRNA expression profiles in various libraries (control, SD and MF radiation), the qualified clean reads were mapped back to human pre-miRNAs (miRBase 19). To eliminate ambiguous multiple hits during the mapping procedure, no mismatch was allowed. Previous studies have reported that, when mapped back to pre-miRNAs, sequence reads typically carry mismatches preferentially located at the terminal $3^{\prime}$ ends (21-24). This mismatch was termed the 3 ' end modification. Based on the read count of all the isomiRs belonging to the same mature miRNAs, the miRNA expression levels were evaluated and presented in transcripts per million (TPM).

Stem-loop reverse transcription and real-time polymerase chain reaction. Reverse transcription ( $\mathrm{RT}$ ) primers were specifically designed for examining miRNAs according to the methods used by Chen et al (25). One microgram of total RNA was reverse transcribed in a stem-loop RT reaction, using RT primers and a SuperScript III Reverse Transcriptase according to the user manual (Invitrogen). The reaction was performed under the following incubation conditions: $30 \mathrm{~min}$ at $16^{\circ} \mathrm{C}$, followed by 50 cycles of $20^{\circ} \mathrm{C}$ for $30 \mathrm{sec}, 42^{\circ} \mathrm{C}$ for $30 \mathrm{sec}$ and $50^{\circ} \mathrm{C}$ for $1 \mathrm{sec}$. The enzyme was subsequently inactivated by incubating it at $85^{\circ} \mathrm{C}$ for $5 \mathrm{~min}$. Real-time polymerase chain reactions (PCRs) were performed using a miRNA-specific 
forward primer and a universal reverse primer combined with incubation at $94^{\circ} \mathrm{C}$ for $10 \mathrm{~min}$, followed by 40 cycles of $94^{\circ} \mathrm{C}$ for $15 \mathrm{sec}$ and $60^{\circ} \mathrm{C}$ for $32 \mathrm{sec}$. The gene expression levels were detected using the SYBR-Green I assay (Applied Biosystems, Foster City, CA, USA), and the miRNA expression levels were normalized to that of U6. The primer sequences for the examined miRNAs are listed as follows: miR-17-RT, CTCAACTG GTGTCGTGGAGTCGGCAATTCAGTTGAGCTACCTGC and miR-17-GSF, CGGCGGCAAAGTGCTTACAGTG; miR18a-RT, CTCAACTGGTGTCGTGGAGTCGGCAATTCAG TTGAGCTATCTGC and miR-18a-GSF, CGGCGGTAAGG TGCATCTAGTG; miR-19a-RT, CTCAACTGGTGTCGTG GAGTCGGCAATTCAGTTGAGTCAGTTTT and miR19aGSF, CGGCGGTGTGCAAATCCATGCA; miR-19b-RT, CTCAACTGGTGTCGTGGAGTCGGCAATTCAGTTGAG TCAGTTTT and miR-19b-GSF, CGGCGGTGTGCAAATC CATGCA; miR-20a-RT, CTCAACTGGTGTCGTGGAGTC GGCAATTCAGTTGAGCTACCTGC and miR-20a-GSF, CGGCGGTAAAGTGCTTATAGTG; miR-92a-RT, CTCAA CTGGTGTCGTGGAGTCGGCAATTCAGTTGAGACAGG CCG and miR-92a-GSF, CGGCGGTATTGCACTTGTCCCG.

miRNA expression level according to The Cancer Genome Atlas data. Members of The Cancer Genome Atlas (TCGA) project collect both cancer and corresponding normal tissues from hundreds of breast cancer patients. All the level-3 miRNA expression data for breast cancer were downloaded from the TCGA data portal (https://tcga-data.nci.nih.gov/tcga/ dataAccessMatrix.htm). These level-3 data included calculated expressions for each miRNA derived from the next-generation sequencing results. In addition, the expression profiles of miRNAs observed in the tumor and corresponding normal samples of 102 patients were downloaded. The normalized quantification expression levels for these 102 participants were further examined for each investigated miRNA.

Pathway enrichment analysis. The target genes of miR-17, miR-18a, miR-19a, miR-19b, miR-20a and miR-92a were downloaded from TargetScan 6.0, and were then mapped onto the Kyoto Encyclopedia of Genes and Genomes (KEGG) pathways based on the Enzyme Commission (EC) numbers by using the R package SubPathwayMiner v.3.1 software (26). Subsequently, a hypergeometric test was performed to identify significantly enriched pathways and to calculate the false positive discovery rate in the FDR-corrected q-value.

\section{Results}

miRNA profiling of radiation-treated breast cancer cells. By using the next-generation sequencing approach, we comprehensively analyzed the distribution of miRNAs in MDA-MB-361 cells after administering SD and MF radiation at 10 Gy. As shown in Table I, we obtained $>8$ million clean reads in 3 libraries. By summarizing the read count of all the isomiRs belonging to the same mature miRNAs, we quantified the miRNA expression abundances, presented in TPM. After mapping the clean reads to the genome, $>500$ miRNAs expressed in the MDA-MB-361 cells $(\mathrm{TPM}>1)$ were detected. Most of these miRNAs were consistently expressed between the control group and the group that received radiation treat-
Table I. Summary of sequences reads information in three libraries.

\begin{tabular}{lcccc}
$\begin{array}{l}\text { Sample } \\
\text { name }\end{array}$ & $\begin{array}{c}\text { Total Illumina } \\
\text { reads }\end{array}$ & $\begin{array}{c}\text { Clean read, } \\
\mathrm{R}>=2\end{array}$ & $\begin{array}{c}\text { Percentage } \\
(\%)\end{array}$ & $\begin{array}{c}\text { Detected } \\
\text { miRs }\end{array}$ \\
\hline Control & $11,229,160$ & $8,741,817$ & 77.85 & 510 \\
MF & $11,086,472$ & $8,780,482$ & 79.20 & 637 \\
SD & $11,685,185$ & $8,982,545$ & 76.87 & 626 \\
\hline
\end{tabular}

We mapped the clean reads back to human pre-miRNAs according to miR-Base 19. MF, multifractionated; SD, single-dose.

ment with SD or MF radiation $\left(\mathrm{R}^{2}>0.8\right.$; Fig. $\left.1 \mathrm{~A}\right)$. Only a small fraction of the miRNAs were identified as being differentially expressed (>1.5-fold) after the MDA-MB-361 cells were exposed to a 10-Gy SD and 2-Gy x 5 fractionated radiation. Comparing the control cells revealed that 13 and 31 miRNAs were upregulated and downregulated, respectively, after undergoing 2-Gy x 5 fractionated radiations. In addition, 52 and 20 miRNAs were upregulated and downregulated, respectively, after undergoing 10-Gy SD radiation (Fig. 1B). Tables II and III list the top 20 upregulated and top 20 downregulated miRNAs after undergoing MF or SD radiation.

$S D$ and $M F$ radiation induces dissimilar miRNA responses in $M D A-M B-361$. Although most of the miRNA expression was consistent after MF and SD radiation treatment $\left(\mathrm{R}^{2}=0.8285\right.$; Fig. 1A), we observed that several radiation-induced miRNA responses were dissimilar between those treated with either $\mathrm{SD}$ or MF radiation. In the present study, we revealed that using various radiation exposure methods caused 8 common miRNAs in MDA-MB-361 cells to change $>1.5$-fold under MF and SD radiation, including the upregulation of 4 miRNAs: miR-32-3p, miR-126-5p, miR-181c-5p and miR-424-5p, and the downregulation of 4 miRNAs: miR-30a-5p, miR-143-3p, miR-339-3p and miR-1246. In addition, the converse response of these miRNAs was frequently observed in the cells that underwent either MF or SD radiation treatment in the present study. The levels of 4 miRNA expressions increased after MF radiation, but after SD radiation, these miRNA expression levels decreased (let-7f-5p, let-7d-5p, miR-423-5p and miR-744-5p). In addition, 16 miRNA expressions were inhibited in 2-Gy MF radiation-treated MDA-MB-361 cells, but were activated following 10-Gy SD radiation treatment.

Radiation-induced miR-17-92 cluster expression. According to the NGS data, we identified several radiation-induced miRNAs and observed that several miRNAs exhibited differential responses to various radiation treatment methods. Among these miRNAs, we observed that the expression levels of miR-19a, miR-20a and miR-19b clearly increased in the MDA-MB-361 cells after undergoing 10-Gy SD radiation treatment (Table II). These miRNAs were located at chromosome 13 and belonged to the miR-17-92 cluster, which has been determined to play an oncogenic role in tumorigenesis by regulating cell survival, proliferation, differentiation and cell cycles (27-29). The miR-17-92 cluster was embedded with 


\section{A}
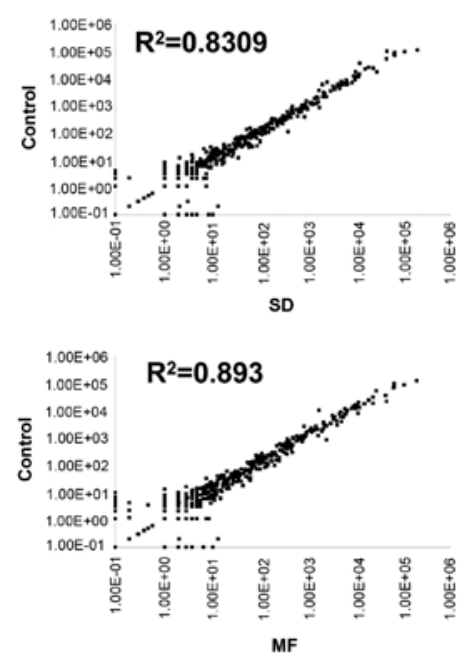

B
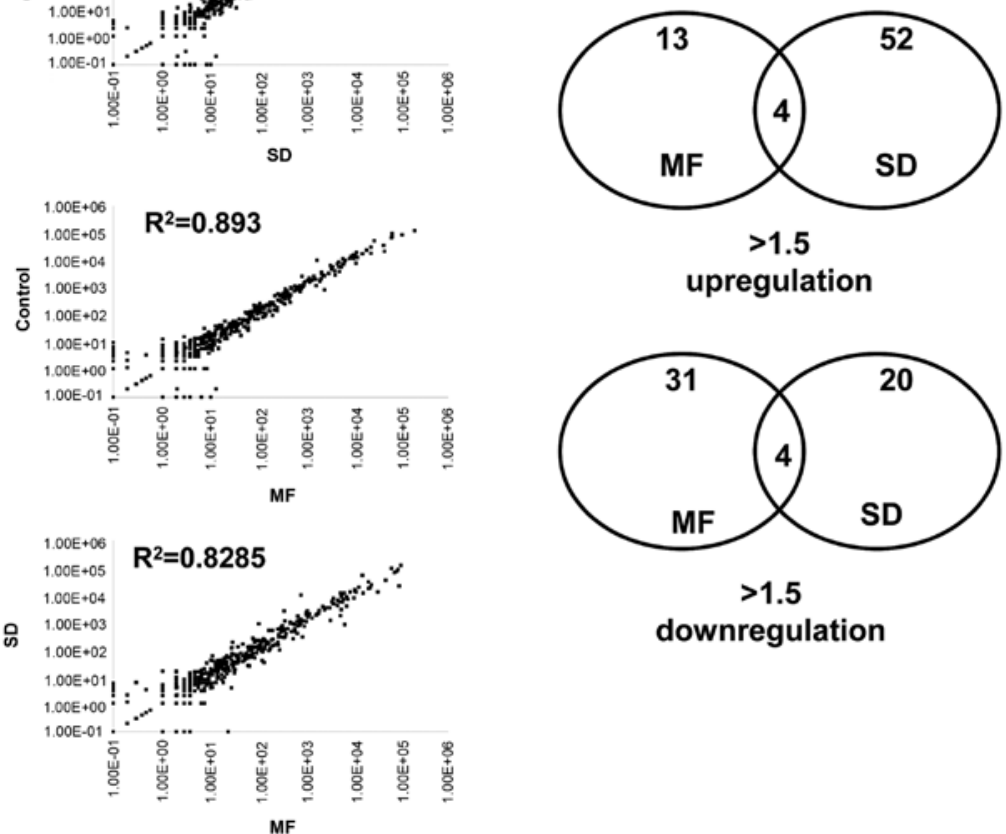

upregulation

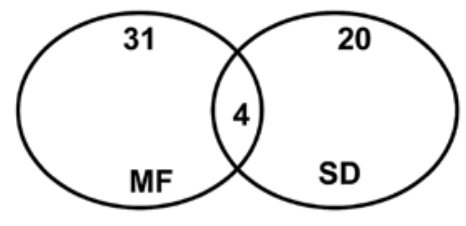

$>1.5$

downregulation

Figure 1. Comparison of miRNA expression profiles in MDA-MB-361 breast cancer cells subjected to single- and multifractionated-dose radiation MDA-MB-361 cells were treated either with or without SD (10 Gy) or MF (5 x 2 Gy) radiation and were subsequently subjected to fresh culture medium. After culturing the samples for an additional $15 \mathrm{~h}$, the miRNA profiles were obtained using next-generation sequencing. (A) Scatter plot of miRNA distribution; SD vs control treatment (top); MF vs. control group (middle) and SD vs. MF (bottom). (B) Venn diagrams depicting the number of upregulated and downregulated miRNAs in MDA-MB-361 after exposure to 10-Gy SD and MF radiation. SD, single-dose; MF, multifractionated.

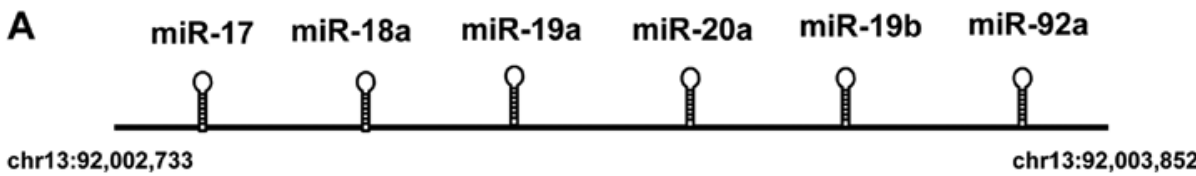

B

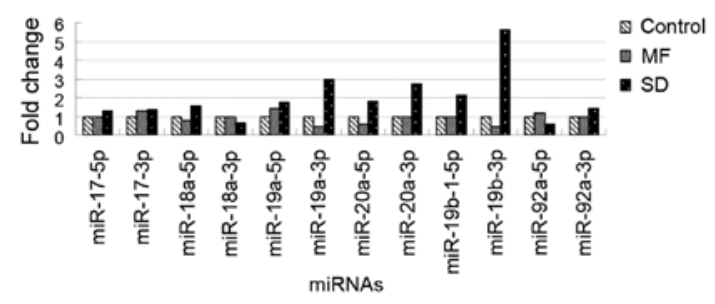

C

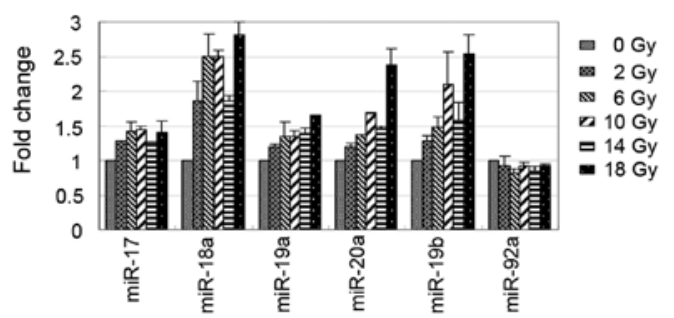

Figure 2. Radiation-induced expression levels of miR-17-92 in breast cancer cells, MDA-MB-361. (A) Schematic displays of the structure and location of the miR-17-92 cluster. (B) Fold changes of the 5p/3p arm of the miR-17-92 cluster after SD and MF radiation treatment were observed using the NGS data. (C) The expression pattern of the miR-17-92 cluster was induced at various radiation treatment doses $(0,2,4,6,8,10,12,14,16$ and 18 Gy) in the MDA-MB-361 cells $\mathrm{SD}$, single-dose; MF, multifractionated. 
Table II. MicroRNAs differentially expressed between control and multi-fractionated radiation.

\begin{tabular}{|c|c|c|c|}
\hline Upregulation & $\begin{array}{l}\text { Control } \\
\text { (RPM) }\end{array}$ & $\begin{array}{c}\mathrm{MF} \\
(\mathrm{RPM})\end{array}$ & $\mathrm{MF} /$ control \\
\hline hsa-let-7e-5p & 2113.1 & 5788.1 & 2.739151 \\
\hline hsa-let-7a-5p & 12845.3 & 34454.3 & 2.68225 \\
\hline hsa-miR-423-5p & 2644.1 & 7037.1 & 2.661435 \\
\hline hsa-let-7f-5p & 47084.2 & 95634.2 & 2.031131 \\
\hline hsa-let-7d-5p & 1791.1 & 3516.1 & 1.963095 \\
\hline hsa-miR-32-5p & 35.1 & 66.1 & 1.883191 \\
\hline hsa-miR-744-5p & 500.1 & 933.1 & 1.865827 \\
\hline hsa-miR-181c-5p & 191.1 & 319.1 & 1.669806 \\
\hline hsa-miR-16-5p & 10650.2 & 16872.2 & 1.584214 \\
\hline hsa-miR-424-3p & 182.1 & 285.1 & 1.565623 \\
\hline hsa-miR-126-3p & 71.1 & 109.1 & 1.534459 \\
\hline hsa-miR-128 & 984.2 & 1473.2 & 1.49685 \\
\hline hsa-miR-361-3p & 85.1 & 127.1 & 1.493537 \\
\hline hsa-miR-320b & 484.2 & 720.2 & 1.487402 \\
\hline hsa-miR-877-5p & 393.1 & 584.1 & 1.485881 \\
\hline hsa-miR-574-5p & 85.1 & 125.1 & 1.470035 \\
\hline hsa-miR-151b & 64.1 & 92.1 & 1.436817 \\
\hline hsa-miR-218-5p & 103.2 & 148.2 & 1.436047 \\
\hline hsa-miR-10a-5p & 3512.1 & 5020.1 & 1.429373 \\
\hline hsa-miR-92b-3p & 5372.1 & 7672.1 & 1.428138 \\
\hline \multicolumn{4}{|l|}{ Downregulation } \\
\hline hsa-miR-1296 & 406.1 & 110.1 & 0.271115 \\
\hline hsa-let-7f-1-3p & 37.1 & 12.1 & 0.326146 \\
\hline hsa-let-7a-3p & 92.2 & 32.2 & 0.349241 \\
\hline hsa-miR-4521 & 74.1 & 31.1 & 0.419703 \\
\hline hsa-miR-19a-3p & 901.1 & 382.1 & 0.424037 \\
\hline hsa-miR-1246 & 108.1 & 47.1 & 0.435708 \\
\hline hsa-miR-19b-3p & 1829.2 & 855.2 & 0.467527 \\
\hline hsa-miR-4286 & 63.1 & 30.1 & 0.477021 \\
\hline hsa-miR-149-5p & 107.1 & 51.1 & 0.477124 \\
\hline hsa-miR-30a-5p & 201261.1 & 105788.1 & 0.525626 \\
\hline hsa-miR-221-3p & 28915.1 & 16665.1 & 0.576346 \\
\hline hsa-miR-4454 & 64.1 & 37.1 & 0.578783 \\
\hline hsa-miR-143-3p & 61.1 & 36.1 & 0.590835 \\
\hline hsa-miR-20a-5p & 644.1 & 388.1 & 0.602546 \\
\hline hsa-miR-339-5p & 158.1 & 105.1 & 0.664769 \\
\hline hsa-miR-598 & 135.1 & 91.1 & 0.674315 \\
\hline hsa-miR-660-5p & 320.1 & 218.1 & 0.68135 \\
\hline hsa-miR-181b-3p & 91.1 & 62.1 & 0.681668 \\
\hline hsa-miR-374b-5p & 60.1 & 41.1 & 0.68386 \\
\hline hsa-miR-561-5p & 203.1 & 140.1 & 0.689808 \\
\hline
\end{tabular}

MF, multifractionated.

6 miRNAs: miR-17, miR-18a, miR-19a, miR-20a, miR-19b and miR-92a (Fig. 2A). As shown in Fig. 2B, the expression levels of most of the miRNAs in the miR-17-92 cluster increased after undergoing SD radiation treatment. Conversely, the
Table III. MicroRNAs differentially expressed between control and single-dose radiation.

\begin{tabular}{|c|c|c|c|}
\hline Upregulation & $\begin{array}{l}\text { Control } \\
\text { (RPM) }\end{array}$ & $\begin{array}{c}\text { SD } \\
(\mathrm{RPM})\end{array}$ & $\mathrm{SD} /$ contro \\
\hline hsa-miR-19b-3p & 1829.2 & 10280.2 & 5.620052 \\
\hline hsa-miR-19a-3p & 901.1 & 2663.1 & 2.955388 \\
\hline hsa-miR-374a-5p & 88.1 & 225.1 & 2.555051 \\
\hline hsa-miR-1285-3p & 118.2 & 267.2 & 2.260575 \\
\hline hsa-miR-1296 & 406.1 & 867.1 & 2.135188 \\
\hline hsa-miR-374b-5p & 60.1 & 128.1 & 2.131448 \\
\hline hsa-miR-454-3p & 242.1 & 511.1 & 2.111111 \\
\hline hsa-miR-210 & 281.1 & 577.1 & 2.053006 \\
\hline hsa-miR-424-5p & 104.1 & 213.1 & 2.04707 \\
\hline hsa-let-7a-3p & 92.2 & 186.2 & 2.019523 \\
\hline hsa-miR-769-5p & 527.1 & 1029.1 & 1.952381 \\
\hline hsa-miR-30c-5p & 6496.2 & 12008.2 & 1.848496 \\
\hline hsa-miR-101-3p & 1258.2 & 2322.2 & 1.845653 \\
\hline hsa-miR-10a-5p & 3512.1 & 6375.1 & 1.815182 \\
\hline hsa-miR-96-5p & 69.1 & 125.1 & 1.81042 \\
\hline hsa-miR-4286 & 63.1 & 114.1 & 1.808241 \\
\hline hsa-miR-221-3p & 28915.1 & 51925.1 & 1.795778 \\
\hline hsa-miR-625-3p & 33.1 & 59.1 & 1.785498 \\
\hline hsa-miR-20a-5p & 644.1 & 1144.1 & 1.776277 \\
\hline hsa-miR-126-5p & 316.1 & 561.1 & 1.775071 \\
\hline \multicolumn{4}{|l|}{ Downregulation } \\
\hline hsa-miR-423-5p & 2644.1 & 847.1 & 0.320374 \\
\hline hsa-let-7f-5p & 47084.2 & 21041.2 & 0.446885 \\
\hline hsa-miR-138-1-3p & 218.1 & 100.1 & 0.458964 \\
\hline hsa-miR-222-5p & 273.1 & 147.1 & 0.538631 \\
\hline hsa-miR-143-3p & 61.1 & 34.1 & 0.558101 \\
\hline hsa-miR-744-5p & 500.1 & 288.1 & 0.576085 \\
\hline hsa-miR-92a-1-5p & 154.1 & 94.1 & 0.610642 \\
\hline hsa-miR-18a-3p & 138.1 & 85.1 & 0.61622 \\
\hline hsa-miR-30a-5p & 201261.1 & 124649.1 & 0.61934 \\
\hline hsa-miR-877-5p & 393.1 & 253.1 & 0.643857 \\
\hline hsa-miR-423-3p & 4484.1 & 2966.1 & 0.661471 \\
\hline hsa-miR-339-3p & 234.1 & 156.1 & 0.666809 \\
\hline hsa-let-7d-5p & 1791.1 & 1195.1 & 0.667244 \\
\hline hsa-miR-30d-5p & 12115.1 & 8090.1 & 0.66777 \\
\hline hsa-miR-1246 & 108.1 & 73.1 & 0.676226 \\
\hline hsa-miR-25-5p & 271.1 & 185.1 & 0.682774 \\
\hline hsa-miR-1275 & 157.1 & 109.1 & 0.694462 \\
\hline hsa-miR-92b-3p & 5372.1 & 3880.1 & 0.722269 \\
\hline hsa-miR-224-5p & 190.1 & 141.1 & 0.742241 \\
\hline hsa-miR-484 & 374.1 & 279.1 & 0.746057 \\
\hline
\end{tabular}

$\mathrm{SD}$, single-dose.

miR-19a-3p, miR-20a-5p and miR-19b-3p expressions were inhibited >1.5-fold in the MDA-MB-361 cells following MF treatment (Fig. 2B). We further examined the expression levels of the miR-17-92 cluster (only the major arm of the miRNAs 


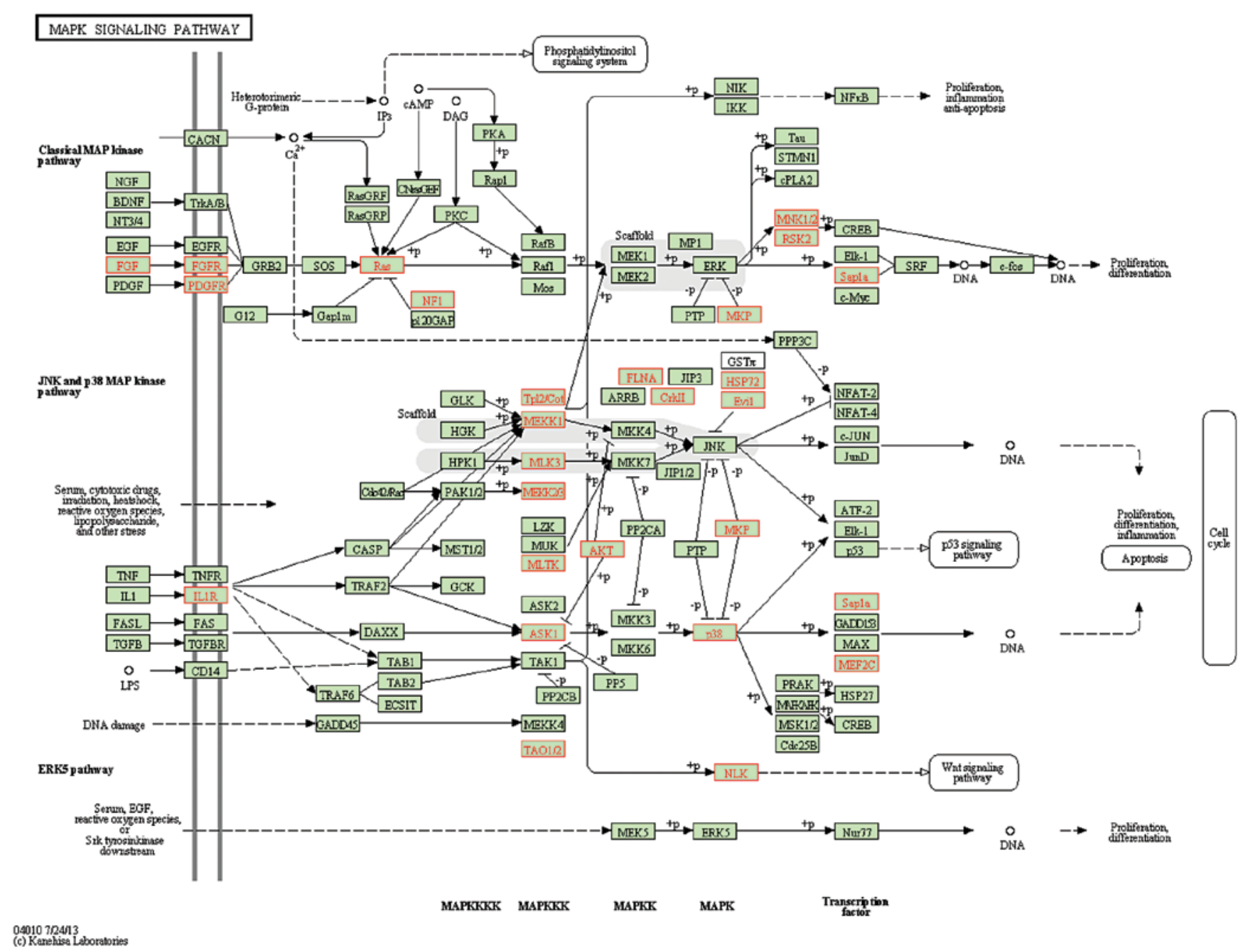

Figure 3. The enriched pathway of the target gene union of the miR-17-92 cluster. (A) The target gene union of the miR-17-92 cluster (miR-17, miR-18a, miR-19a/b, miR-20a and miR-92a) enriched in the MAPK pathway (FDR=1.1E-8). The target genes are labeled in red.

was selected) in the MDA-MB-361 cells following treatment with various doses of radiation for $15 \mathrm{~h}$, using the real-time PCR approach. As shown in Fig. 2C, the expression levels of the miRNAs in the miR-17-92 cluster gradually increased in a dose-dependent manner $(0,2,6,10,14$ and $18 \mathrm{~Gy})$. These results indicated that, except for miR-92a, miR-17-92 cluster expression can be activated by radiation treatment in MDA-MB-361 cells.

Analysis of the role of the miR-17-92 cluster in breast cancer by using an in silico approach. Generally, one miRNA typically contains hundreds of target genes and slightly suppresses its own target gene; therefore, conducting a biological function by using a group of miRNAs comodulated with the same signaling pathway may be more efficient. According to this theory, we further examined the miR-17-92 cluster function by using pathway enrichment analysis. We first obtained the putative target genes of a miR-17-92 cluster from TargetScan 6.0; subsequently, these target genes were mapped onto KEGG pathways. The data indicated that the target genes of the miR-17-92 cluster were frequently and significantly enriched in several cancer-related and radiation-related pathways, including the mitogen-activated protein kinase (MAPK), ErbB, p53, Wnt, transforming growth factor- $\beta$ (TGF- $\beta$ ) and mTOR signaling pathways, and cell cycle with an FDR $<0.05$ (Table IV; Fig. 3). These data indicated that the miR-17-92 cluster participated in cancer cell progression and played a crucial role in radiation therapy by regulating radiation-response pathways.

To further elucidate the role of the miR-17-92 cluster in breast cancer, we analyzed the expression levels of a miR-17-92 cluster in breast cancer, which were provided by the TCGA dataset, using in silico analysis. We downloaded 204 miRNA expression profiles characterizing 102 breast cancer patients. These profiles included breast cancer lesions and the corresponding normal tissues. As shown in Fig. 4A, the expression levels of miR-17, miR-18a, miR-19a, miR-20a and miR-19b were significantly upregulated in the breast cancer cells compared with those of the corresponding normal tissue cells. miR-92a expression was significantly decreased in the breast cancer cells compared with that of the corresponding adjacent cells. We also further examined the expression levels of miR-19a, miR-19b, miR-20a and miR-92a by using real-time PCR in the breast cancer cells. These results were consistent with the TCGA data, indicating that miR-19a and miR-19b expression is significantly upregulated and miR-92a is downregulated in breast cancer cells compared with that of adjacent 
Table IV. Predicted targets of miR-17-92 cluster involved in radiation-relative pathways.

\begin{tabular}{|c|c|c|c|}
\hline Pathway & AnnMolecule ratio & AnnBg ratio & FDR \\
\hline Endocytosis & $67 / 2785$ & $203 / 21796$ & $1.21 \mathrm{E}-11$ \\
\hline MAPK signaling pathway ${ }^{a}$ & $73 / 2785$ & $266 / 21796$ & $1.10 \mathrm{E}-08$ \\
\hline Axon guidance & $42 / 2785$ & $126 / 21796$ & $1.38 \mathrm{E}-07$ \\
\hline Pathways in cancer ${ }^{\mathrm{a}}$ & $78 / 2785$ & $322 / 21796$ & $6.70 \mathrm{E}-07$ \\
\hline mTOR signaling pathway ${ }^{\mathrm{a}}$ & $22 / 2785$ & $52 / 21796$ & $5.06 \mathrm{E}-06$ \\
\hline Neurotrophin signaling pathway & $37 / 2785$ & $126 / 21796$ & 2.37E-05 \\
\hline Regulation of actin cytoskeleton & $51 / 2785$ & $211 / 21796$ & 0.000122 \\
\hline Melanogenesis & $30 / 2785$ & $100 / 21796$ & 0.000122 \\
\hline Focal adhesion & $48 / 2785$ & $199 / 21796$ & 0.000188 \\
\hline GnRH signaling pathway & $29 / 2785$ & $98 / 21796$ & 0.000188 \\
\hline Glioma & $22 / 2785$ & $65 / 21796$ & 0.000196 \\
\hline Long-term potentiation & $23 / 2785$ & $70 / 21796$ & 0.000199 \\
\hline Dilated cardiomyopathy & $26 / 2785$ & $85 / 21796$ & 0.000217 \\
\hline Melanoma & $23 / 2785$ & $71 / 21796$ & 0.000222 \\
\hline Chronic myeloid leukemia & $23 / 2785$ & $72 / 21796$ & 0.000268 \\
\hline Wnt signaling pathway ${ }^{a}$ & $38 / 2785$ & $150 / 21796$ & 0.000285 \\
\hline Renal cell carcinoma & $22 / 2785$ & $68 / 21796$ & 0.000285 \\
\hline Progesterone-mediated oocyte maturation & $26 / 2785$ & $88 / 21796$ & 0.000309 \\
\hline Pancreatic cancer & $22 / 2785$ & $70 / 21796$ & 0.000423 \\
\hline Calcium signaling pathway & $42 / 2785$ & $177 / 21796$ & 0.000487 \\
\hline Phosphatidylinositol signaling system ${ }^{a}$ & $23 / 2785$ & $78 / 21796$ & 0.000787 \\
\hline Oocyte meiosis & $29 / 2785$ & $111 / 21796$ & 0.001083 \\
\hline Salivary secretion & $24 / 2785$ & $86 / 21796$ & 0.00132 \\
\hline TGF- $\beta$ signaling pathway ${ }^{a}$ & $23 / 2785$ & $82 / 21796$ & 0.001591 \\
\hline Prostate cancer & $24 / 2785$ & $88 / 21796$ & 0.001777 \\
\hline Small cell lung cancer & $23 / 2785$ & $83 / 21796$ & 0.001777 \\
\hline Hypertrophic cardiomyopathy (HCM) & $22 / 2785$ & $78 / 21796$ & 0.001777 \\
\hline Bladder cancer & $14 / 2785$ & $40 / 21796$ & 0.002097 \\
\hline Adipocytokine signaling pathway & $19 / 2785$ & $65 / 21796$ & 0.002643 \\
\hline ErbB signaling pathway ${ }^{a}$ & $23 / 2785$ & $86 / 21796$ & 0.002728 \\
\hline Insulin signaling pathway & $32 / 2785$ & $136 / 21796$ & 0.002821 \\
\hline Ubiquitin mediated proteolysis & $32 / 2785$ & $137 / 21796$ & 0.003136 \\
\hline Non-small cell lung cancer & $16 / 2785$ & $54 / 21796$ & 0.005639 \\
\hline Circadian rhythm-mammal & $9 / 2785$ & $22 / 21796$ & 0.005986 \\
\hline Gastric acid secretion & $19 / 2785$ & $72 / 21796$ & 0.00868 \\
\hline p53 signaling pathway ${ }^{\mathrm{a}}$ & $18 / 2785$ & $67 / 21796$ & 0.008943 \\
\hline Acute myeloid leukemia & $16 / 2785$ & $57 / 21796$ & 0.009507 \\
\hline Colorectal cancer & $17 / 2785$ & $63 / 21796$ & 0.010764 \\
\hline Cell cycle ${ }^{a}$ & $28 / 2785$ & $127 / 21796$ & 0.014135 \\
\hline Vasopressin-regulated water reabsorption & $13 / 2785$ & $44 / 21796$ & 0.014273 \\
\hline Dorso-ventral axis formation & $9 / 2785$ & $26 / 21796$ & 0.019367 \\
\hline Inositol phosphate metabolism & $15 / 2785$ & $57 / 21796$ & 0.022744 \\
\hline Vascular smooth muscle contraction & $25 / 2785$ & $114 / 21796$ & 0.022744 \\
\hline Aldosterone-regulated sodium reabsorption & $12 / 2785$ & $42 / 21796$ & 0.025281 \\
\hline Fc $\varepsilon$ RI signaling pathway & $18 / 2785$ & $75 / 21796$ & 0.026074 \\
\hline Hepatitis C & $28 / 2785$ & $134 / 21796$ & 0.026074 \\
\hline Amyotrophic lateral sclerosis (ALS) & $14 / 2785$ & $53 / 21796$ & 0.026074 \\
\hline Fc $\gamma$ R-mediated phagocytosis & $20 / 2785$ & $87 / 21796$ & 0.027332 \\
\hline Arrhythmogenic right ventricular cardiomyopathy (ARVC) & $17 / 2785$ & $71 / 21796$ & 0.031624 \\
\hline Chemokine signaling pathway & $36 / 2785$ & $187 / 21796$ & 0.032834 \\
\hline
\end{tabular}


Table IV. Continued.

\begin{tabular}{|c|c|c|c|}
\hline Pathway & AnnMolecule ratio & AnnBg ratio & FDR \\
\hline Glycosphingolipid biosynthesis-ganglio series & $6 / 2785$ & $15 / 21796$ & 0.03323 \\
\hline ECM-receptor interaction & $19 / 2785$ & $84 / 21796$ & 0.036636 \\
\hline Hedgehog signaling pathway & $14 / 2785$ & $56 / 21796$ & 0.038697 \\
\hline
\end{tabular}

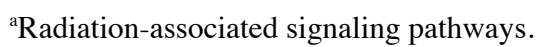

A
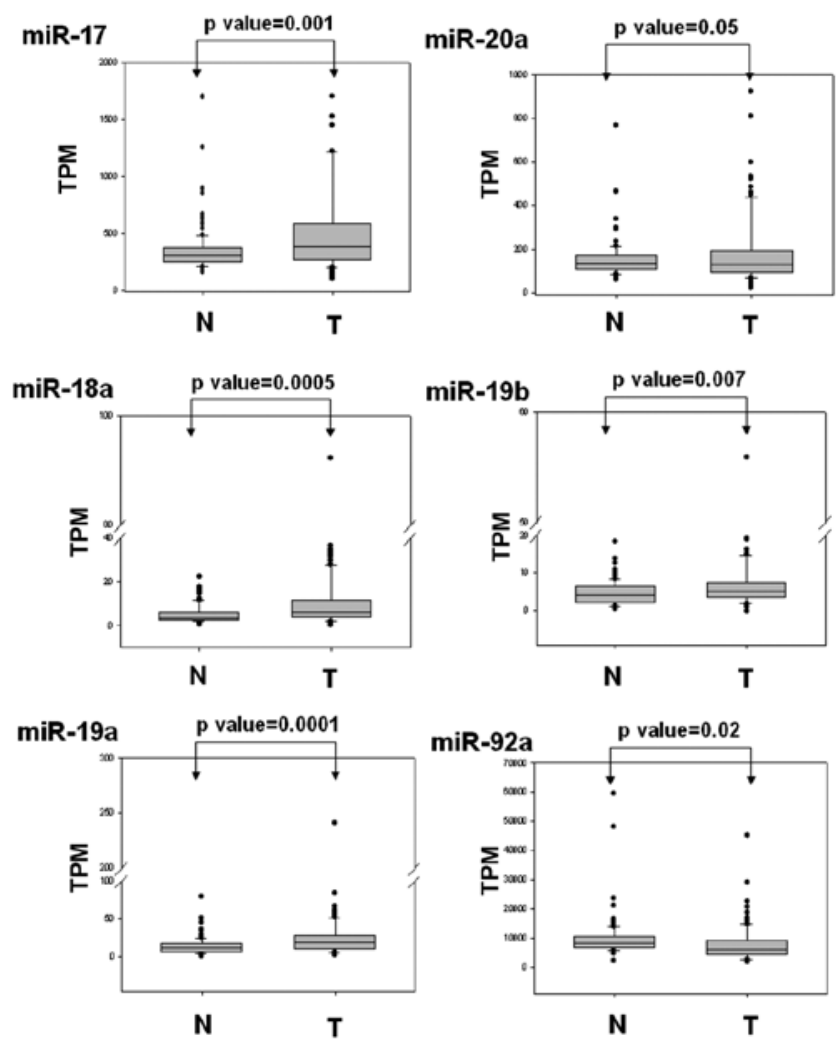

normal tissues (Fig. 4B). Collectively, the data indicated that expression levels of the miR-17-92 cluster were deregulated in breast cancer cells, suggesting that the miR-17-92 cluster plays a vital role in breast cancer progression.

\section{Discussion}

miRNAs modulate gene expression by degrading mRNA and inhibiting protein translation, and thereby contribute to the regulation of numerous cancer-relevant cellular phenotypes, including cell proliferation, apoptosis, cell cycle, cell motility and stress response $(30,31)$. Moreover, dysfunctional miRNAs are involved in breast cancer carcinogenesis through the impairment of cancer-related pathways (32). Understanding the functions of miRNAs may contribute to cancer diagnosis, prognosis and therapy $(33,34)$. Previous studies have indicated that several miRNAs can serve as biomarkers for the diagnosis and prognosis of breast cancer (35). In a previous study, the expression of miR-210 was determined to be associated

\section{B}
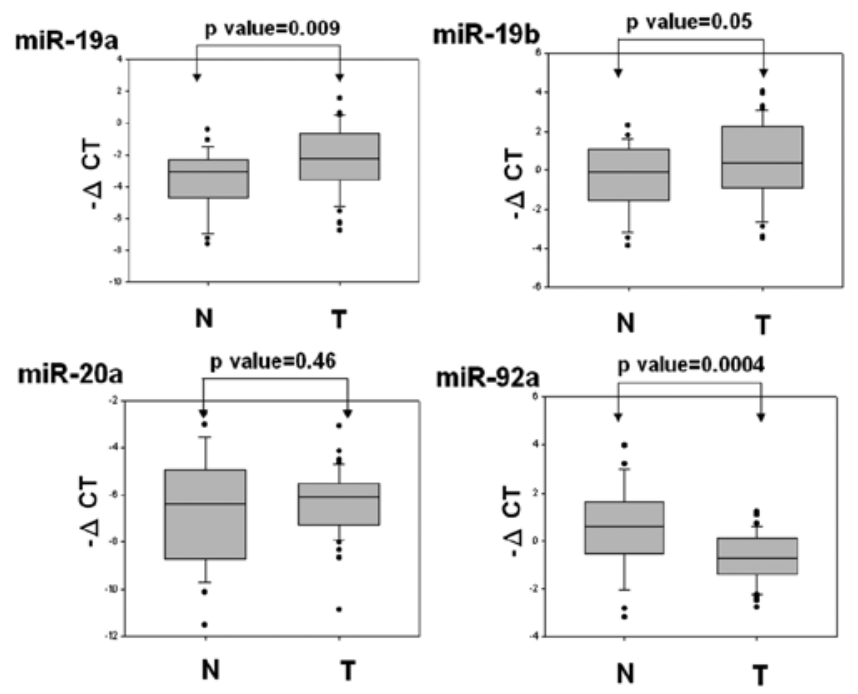

Figure 4. Expression levels of the miR-17-92 cluster in breast cancer cells. Comparison of the miRNA expression levels between the tumor and corresponding normal tissues obtained from 102 breast cancer patients, which were analyzed using the TCGA dataset. The expression levels of the miRNAs were presented in transcripts per million (TPM). (B) The expression levels of miR-19a/b, miR-20a and miR-92a in clinical breast cancer samples were analyzed using quantitative stem-loop PCR with U6 as an internal control. All the samples were assessed in triplicate and analyzed using the Student's t-test $(\mathrm{P}<0.05$ was considered significant).

with the clinical prognosis of breast cancer. The upregulation of miR-210 was reported to contribute to decreased rates of disease-free and overall survival (36). Kovalchuk et al (37) revealed that miR-451 is correlated with the expression of multidrug resistant genes and that drug sensitivity to doxorubicin-resistant breast cancer could be enhanced by restoring miR-451 into the cell. Thus, correcting the altered expression of miRNA by restoring miR-451 can also be used as a strategy to improve responsiveness to chemotherapy. In addition, miRNAs were observed to exhibit high stability in human serum and plasma (38). One recent study demonstrated that a decrease in the levels of miR-92a and an increase in the levels of miR-21 were consistent and specific in the serum of breast cancer patients, and suggested that miRNA can be used as biomarkers for early breast cancer detection in the future (39).

Radiation-induced DNA damage initiates cellular responses, such as cell cycle arrest, DNA damage repair and apoptosis (40). Previous studies have revealed that miRNAs also act as potential agents for predicting radiation responses or 
are used to modulate tumor radiation response to cancer cells further, thus enhancing the efficacy of radiation therapy against resistant cancers $(34,35,40)$. In breast cancer, several miRNAs have been identified as critical in influencing the radiosensitivity of breast cancer during radiation therapy, including let-7 family miR-7, miR-21, miR-31, miR-200c, miR-199a and miR-302a (13-18). Among the miRNAs identified as potential agents that contribute to radiation responsiveness, let-7 family was one of the first miRNAs to be investigated. A previous study revealed that let-7a overexpression can suppress the expression of K-Ras and radiosensitize lung cancer cells (41). Another study determined that the ectopic overexpression of miR-7 enhanced EGFR and Akt expression and radiosensitized laryngeal, breast and lung cancer cell (14). In addition, a recent study demonstrated that miR-21 expression in breast cancer cells contributes to radiation resistance by inhibiting the $G 2 / M$ check point (16). The upregulation of miR-302a sensitizes radioresistant breast cancer cells and reduces the expression of AKT1 and RAD52 (42). Furthermore, miR-199a-5p suppresses radiation-induced autophagy, and inhibits DRAM1 and Beclin1 expression in breast cancer cells (17).

The miR-17-92 cluster is an oncogenic miRNA that regulates cell survival, proliferation, differentiation and angiogenesis in most human types of cancer, including breast cancer (43-45). Previous studies revealed that the overexpression of miR-17-92 in human cancer cells markedly decreases the radiosensitivity of these cells through the repression of PTEN and PHLPP2, which causes the activation of the PI3K/AKT pathway to become enhanced (46). In the present study, the data revealed that the numerous miRNA responses differed between the cells that underwent either SD or MF radiation. Among the miRNAs, we observed that the expressions of the miR-17-92 cluster were upregulated under SD radiation treatment. Conversely, these expressions were frequently inhibited following MF radiation treatment (Fig. 2B). Tsai et al (6) reported that the survival rate of breast cancer cells following MF (5 x 2 Gy) was higher than that following $\mathrm{SD}$, which was expected since sublethal damage was repaired between fractions. Exposing the breast cancer cells to SD at 10 Gy led to the predominant entry of these cells into the cell death pathway. The observation that the cells were affected differently following SD and MF radiation treatment could explain why miRNA expressions frequently vary based on the treatment method used. John-Aryankalayil et al (20) also demonstrated the differential expression patterns of several miRNAs, including miR-17-92 cluster, miR-34a, let-7 family miRNAs and has-miR-146a, in 3 prostate cancer cell lines after undergoing SD and fractionated radiation. Their results revealed that the miR-17-92 cluster is markedly downregulated by radiation treatment in $\mathrm{p} 53$-positive prostate cells. Following radiation treatment, increasing wild-type p53 expression causes cells to accumulate in the G1 phase (47). The status of p53 was demonstrated to significantly influence the expression profile of genes following fractionated irradiation (48). Since a mutated p53 gene cell line, MDA-MB-361, was used in the present study, p53-regulated genes should not be induced in MDA-MB-361 cells following radiation treatment. The elevated expression levels of a miR-17-92 cluster in MDA-MB-361 cells undergoing radiation treatment should be p53-independent pathway. Yan et al (49) reported that the
miR-17-92 cluster is a novel target for p53-mediated transcriptional repression. The transcription factors, c-Myc and E2F family, have been observed to directly bind to the promoter of the miR-17-92 cluster and increase the transcriptional activity of the cluster (50-52). Previous studies have reported that expression levels of c-Myc and E2F can be induced in breast cancer cells following radiation treatment (53-55). According to these studies, cells with wild-type p53 cause the expression levels of the miR-17-92 cluster to decrease through radiationinduced p53 activity. Conversely, an increase in miR-17-92 transcriptional activity results from c-Myc or E2F expression levels in mutated p53 cells undergoing radiation treatment.

In the present study, we identified several radiation-associated miRNAs that were clearly altered under either SD or MF radiation treatment, including let-7 family, miR-138 and the miR-17-92 cluster ( $>2$-fold changes; Tables II and III). Wang et al (13) demonstrated that overexpression of Let-7 enhanced the sensitivity of breast cancer cells to radiation. In addition, miR-138 expression increased under SD treatment. However, overexpression of miR-138 inhibited homologous recombination and enhanced the sensitivity of osteosarcoma cells to multiple DNA-damaging agents including radiation (56). Niemoeller et al (57) identified the expression levels of numerous miRNAs known to be involved in the regulation of cellular processes such as apoptosis, proliferation, invasion, local immune response and radioresistance. These miRNAs, including let-7, miR-138 and miR-1285, displayed 2-3-fold changes after undergoing irradiation. Moreover, several miRNAs previously not known to be radiation responsive were determined in the present study, including miR-149, miR-374a/b, miR-423, miR-424, miR-454, miR-1246 and miR-1296. These miRNA candidates can serve as effective targets for improving the efficacy of radiation treatment in future breast cancer therapy.

\section{Acknowledgements}

This study was supported by grants from the Kaohsiung Veterans General Hospital (VGHKS 103-G01-3 and VGHKS 103-043). The authors would like to thank Genomics and Proteomics Core Laboratory, Department of Medical Research, Kaohsiung Chang Gung Memorial Hospital, for the help with NGS data analysis.

\section{References}

1. Delaney G, Jacob S, Featherstone C and Barton M: The role of radiotherapy in cancer treatment: estimating optimal utilization from a review of evidence-based clinical guidelines. Cancer 104: 1129-1137, 2005.

2. Rzeszowska-Wolny J, Przybyszewski WM and Widel M: Ionizing radiation-induced bystander effects, potential targets for modulation of radiotherapy. Eur J Pharmacol 625: 156-164, 2009.

3. Runkle EA, Zhang H, Cai Z, et al: Reversion of the ErbB malignant phenotype and the DNA damage response. Exp Mol Pathol 93: 324-333, 2012.

4. Yin E, Nelson DO, Coleman MA, Peterson LE and Wyrobek AJ: Gene expression changes in mouse brain after exposure to low-dose ionizing radiation. Int J Radiat Biol 79: 759-775, 2003.

5. Correa CR and Cheung VG: Genetic variation in radiation-induced expression phenotypes. Am J Hum Genet 75: 885-890, 2004.

6. Tsai MH, Cook JA, Chandramouli GV, et al: Gene expression profiling of breast, prostate, and glioma cells following single versus fractionated doses of radiation. Cancer Res 67: 3845-3852, 2007. 
7. John-Aryankalayil M, Palayoor ST, Cerna D, et al: Fractionated radiation therapy can induce a molecular profile for therapeutic targeting. Radiat Res 174: 446-458, 2010.

8. Bartel DP: MicroRNAs: genomics, biogenesis, mechanism, and function. Cell 116: 281-297, 2004.

9. Krol J, Loedige I and Filipowicz W: The widespread regulation of microRNA biogenesis, function and decay. Nat Rev Genet 11: 597-610, 2010

10. Iorio MV, Ferracin M, Liu CG, et al: MicroRNA gene expression deregulation in human breast cancer. Cancer Res 65: 7065-7070, 2005 .

11. Guo X, Wu Y and Hartley RS: MicroRNA-125a represses cell growth by targeting HuR in breast cancer. RNA Biol 6: 575-583, 2009.

12. Ma L, Teruya-Feldstein $\mathrm{J}$ and Weinberg RA: Tumour invasion and metastasis initiated by microRNA-10b in breast cancer. Nature 449: 682-688, 2007.

13. Wang L, Yuan C, Lv K, et al: Lin 28 mediates radiation resistance of breast cancer cells via regulation of caspase, H2A.X and Let-7 signaling. PLoS One 8: e67373, 2013.

14. Lee KM, Choi EJ and Kim IA: microRNA-7 increases radiosensitivity of human cancer cells with activated EGFR-associated signaling. Radiother Oncol 101: 171-176, 2011.

15. Lin J, Liu C, Gao F, et al: miR-200c enhances radiosensitivity of human breast cancer cells. J Cell Biochem 114: 606-615, 2013.

16. Anastasov N, Höfig I, Vasconcellos IG, et al: Radiation resistance due to high expression of miR-21 and G2/M checkpoint arrest in breast cancer cells. Radiat Oncol 7: 206, 2012.

17. Yi H, Liang B, Jia J, et al: Differential roles of miR-199a-5p in radiation-induced autophagy in breast cancer cells. FEBS Lett 587: 436-443, 2013.

18. Körner C, Keklikoglou I, Bender C, Wörner A, Münstermann E, and Wiemann S: MicroRNA-31 sensitizes human breast cells to apoptosis by direct targeting of protein kinase $\mathrm{C} \varepsilon(\mathrm{PKC} \varepsilon)$. J Biol Chem 288: 8750-8761, 2013.

19. Cloonan N, Wani S, Xu Q, et al: MicroRNAs and their isomiRs function cooperatively to target common biological pathways. Genome Biol 12: R126, 2011

20. John-Aryankalayil M, Palayoor ST, Makinde AY, et al: Fractionated radiation alters oncomir and tumor suppressor miRNAs in human prostate cancer cells. Radiat Res 178: 105-117, 2012.

21. Ebhardt HA, Tsang HH, Dai DC, Liu Y, Bostan B and Fahlman RP: Meta-analysis of small RNA-sequencing errors reveals ubiquitous post-transcriptional RNA modifications. Nucleic Acids Res 37 2461-2470, 2009.

22. Landgraf $\mathrm{P}$, Rusu M, Sheridan R, et al: A mammalian microRNA expression atlas based on small RNA library sequencing. Cell 129: 1401-1414, 2007.

23. Reid JG, Nagaraja AK, Lynn FC, et al: Mouse let-7 miRNA populations exhibit RNA editing that is constrained in the 5'-seed/ cleavage/anchor regions and stabilize predicted $\mathrm{mmu}-$ let-7a:mRNA duplexes. Genome Res 18: 1571-1581, 2008

24. Morin RD, O'Connor MD, Griffith M, et al: Application of massively parallel sequencing to microRNA profiling and discovery in human embryonic stem cells. Genome Res 18 610-621, 2008

25. Chen C, Ridzon DA, Broomer AJ, et al: Real-time quantification of microRNAs by stem-loop RT-PCR. Nucleic Acids Res 33: e179, 2005

26. Li C, Li X, Miao Y, et al: SubpathwayMiner: a software package for flexible identification of pathways. Nucleic Acids Res 37: e131, 2009 .

27. He L, Thomson JM, Hemann MT, et al: A microRNA polycistron as a potential human oncogene. Nature 435: 828-833, 2005.

28. Hayashita Y, Osada H, Tatematsu Y, et al: A polycistronic microRNA cluster, $m i R-17-92$, is overexpressed in human lung cancers and enhances cell proliferation. Cancer Res 65 9628-9632, 2005.

29. Cho WC: OncomiRs: the discovery and progress of microRNAs in cancers. Mol Cancer 6: 60, 2007.

30. Pan HW, Li SC and Tsai KW: MicroRNA dysregulation in gastric cancer. Curr Pharm Des 19: 1273-1284, 2013.

31. Zhao L, Bode AM, Cao Y and Dong Z: Regulatory mechanisms and clinical perspectives of miRNA in tumor radiosensitivity. Carcinogenesis 33: 2220-2227, 2012.

32. Chang HT, Li SC, Ho MR, et al: Comprehensive analysis of microRNAs in breast cancer. BMC Genomics 13 (Suppl 7): S18, 2012.

33. Ferracin M, Querzoli P, Calin GA and Negrini M: MicroRNAs: toward the clinic for breast cancer patients. Semin Oncol 38 : 764-775, 2011
34. Ng EK, Wong CL, Ma ES and Kwong A: MicroRNAs as new players for diagnosis, prognosis, and therapeutic targets in breast cancer. J Oncol 2009: 305420, 2009.

35. Iorio MV, Casalini P, Piovan C, Braccioli L and Tagliabue E: Breast cancer and microRNAs: therapeutic impact. Breast 20 (Suppl 3): S63-S70, 2011.

36. Camps C, Buffa FM, Colella S, et al: hsa-miR-210 is induced by hypoxia and is an independent prognostic factor in breast cancer. Clin Cancer Res 14: 1340-1348, 2008.

37. Kovalchuk O, Filkowski J, Meservy J, et al: Involvement of microRNA-451 in resistance of the MCF-7 breast cancer cells to chemotherapeutic drug doxorubicin. Mol Cancer Ther 7: 2152-2159, 2008

38. Mitchell PS, Parkin RK, Kroh EM, et al: Circulating microRNAs as stable blood-based markers for cancer detection. Proc Natl Acad Sci USA 105: 10513-10518, 2008.

39. Si H, Sun X, Chen Y, et al: Circulating microRNA-92a and microRNA-21 as novel minimally invasive biomarkers for primary breast cancer. J Cancer Res Clin Oncol 139: 223-229, 2013.

40. Zhao L, Lu X and Cao Y: MicroRNA and signal transduction pathways in tumor radiation response. Cell Signal 25: 1625-1634, 2013.

41. Weidhaas JB, Babar I, Nallur SM, et al: MicroRNAs as potential agents to alter resistance to cytotoxic anticancer therapy. Cancer Res 67: 11111-11116, 2007.

42. Liang Z, Ahn J, Guo D, Votaw JR and Shim H: MicroRNA-302 replacement therapy sensitizes breast cancer cells to ionizing radiation. Pharm Res 30: 1008-1016, 2013.

43. de Rinaldis E, Gazinska P, Mera A, et al: Integrated genomic analysis of triple-negative breast cancers reveals novel microRNAs associated with clinical and molecular phenotypes and sheds light on the pathways they control. BMC Genomics 14: 643, 2013.

44. Kim K, Chadalapaka G, Lee SO, et al: Identification of oncogenic microRNA-17-92/ZBTB4/specificity protein axis in breast cancer. Oncogene 31: 1034-1044, 2012.

45. Li H, Bian C, Liao L, Li J and Zhao RC: miR-17-5p promotes human breast cancer cell migration and invasion through suppression of HBP1. Breast Cancer Res Treat 126: 565-575, 2011.

46. Jiang P, Rao EY, Meng N, Zhao Y and Wang JJ: MicroRNA17-92 significantly enhances radioresistance in human mantle cell lymphoma cells. Radiat Oncol 5: 100, 2010.

47. Kuerbitz SJ, Plunkett BS, Walsh WV and Kastan MB: Wild-type p53 is a cell cycle checkpoint determinant following irradiation. Proc Natl Acad Sci USA 89: 7491-7495, 1992.

48. Simone CB II, John-Aryankalayil M, Palayoor ST, et al: mRNA expression profiles for prostate cancer following fractionated irradiation are influenced by p53 status. Transl Oncol 6: 573-585, 2013.

49. Yan HL, Xue G, Mei Q, et al: Repression of the miR-17-92 cluster by 53 has an important function in hypoxia-induced apoptosis. EMBO J 28: 2719-2732, 2009.

50. Thomas M, Lange-Grunweller K, Hartmann D, et al: Analysis of transcriptional regulation of the human miR-17-92 cluster; evidence for involvement of Pim-1. Int J Mol Sci 14: 12273-12296, 2013.

51. Li Y, Li Y, Zhang H and Chen Y: MicroRNA-mediated positive feedback loop and optimized bistable switch in a cancer network involving miR-17-92. PLoS One 6: e26302, 2011.

52. Aguda BD, Kim Y, Piper-Hunter MG, Friedman A and Marsh CB MicroRNA regulation of a cancer network: consequences of the feedback loops involving miR-17-92, E2F, and Myc. Proc Natl Acad Sci USA 105: 19678-19683, 2008

53. Papanikolaou V, Iliopoulos D, Dimou I, et al: Survivin regulation by HER 2 through NF- $\kappa$ B and c-myc in irradiated breast cancer cells. J Cell Mol Med 15: 1542-1550, 2011.

54. Orr MS, Watson NC, Sundaram S, Randolph JK, Jain PT and Gewirtz DA: Ionizing radiation and teniposide increase p21 $1^{\text {waf } 1 / c i p 1}$ and promote $\mathrm{Rb}$ dephosphorylation but fail to suppress E2F activity in MCF-7 breast tumor cells. Mol Pharmacol 52: 373-379, 1997.

55. Calaf GM and Hei TK: Ionizing radiation induces alterations in cellular proliferation and c-myc, c-jun and c-fos protein expression in breast epithelial cells. Int J Oncol 25: 1859-1866, 2004.

56. Wang Y, Huang JW, Li M, et al: MicroRNA-138 modulates DNA damage response by repressing histone H2AX expression. Mol Cancer Res 9: 1100-1111, 2011.

57. Niemoeller OM, Niyazi M, Corradini S, et al: MicroRNA expression profiles in human cancer cells after ionizing radiation. Radiat Oncol 6: 29, 2011. 